\title{
UNSTEADY HARTMANN TWO-PHASE FLOW: THE RIEMANN-SUM APPROXIMATION APPROACH
}

\author{
B.K. JHA and C.T. BABILA \\ Department of Mathematics, Ahmadu Bello University \\ Zaria, NIGERIA \\ E-mail: basant777@yahoo.com \\ chrisbabisco@yahoo.com \\ S. ISA ${ }^{*}$ \\ Department of Mathematics, Yobe State University \\ Damaturu, NIGERIA \\ E-mail: sanimath@yahoo.com
}

\begin{abstract}
We consider the time dependent Hartmann flow of a conducting fluid in a channel formed by two horizontal parallel plates of infinite extent, there being a layer of a non-conducting fluid between the conducting fluid and the upper channel wall. The flow formation of conducting and non-conducting fluids is coupled by equating the velocity and shear stress at the interface. The unsteady flow formation inside the channel is caused by a sudden change in the pressure gradient. The relevant partial differential equations capturing the present physical situation are transformed into ordinary differential equations using the Laplace transform technique. The ordinary differential equations are then solved analytically and the Riemann-sum approximation method is used to invert the Laplace domain into time domain. The solution obtained is validated by comparisons with the closed form solutions obtained for steady states which have been derived separately and also by the implicit finite difference method. The variation of velocity, mass flow rate and skin-friction on both plates for various physical parameters involved in the problem are reported and discussed with the help of line graphs. It was found that the effect of changes of the electric load parameter is to aid or oppose the flow as compared to the short-circuited case.
\end{abstract}

Key words: Hartmann two-phase flow, unsteady flow, Riemann-sum approximation.

\section{Introduction}

The occurrences of stratified two-phase flows in petroleum industry and in many engineering processes have prompted much interest in both theoretical and experimental studies on a stratified laminar flow of two immiscible fluids in different geometry. There have been quite a few experimental and analytical studies on hydrodynamic aspects of the two-phase flow reported in the recent literature. The interest in these types of problems stems from the possibility of reducing the power required to pump oil in a pipeline by a suitable addition of water.

Shail [1] studied the Hartmann flow of a conducting fluid in a channel between two horizontal insulating plates of infinite extent with a layer of a non-conducting fluid between the upper wall and the conducting fluid. He observed that an increase of order $30 \%$ could be achieved in the flow rate for suitable ratios of heights and viscosities of the two fluids. The Hartmann flow past a permeable bed in the presence of a transverse magnetic field with an interface at the surface of the permeable bed was presented by Rudraiah et al. [2]. A steady MHD channel flow has been presented in the pioneering work of Hartmann [3, 4]. Realizing the physical importance of such physical situations, Malashetty and Leela [5] and Lohrasbi and

\footnotetext{
* To whom correspondence should be addressed
} 
Sahai [6] studied the two-phase MHD flow and heat transfer in a parallel plate channel, where both phases are incompressible and the flow is assumed to be steady, one-dimensional and fully developed. These studies constituted an attempt to provide a clear understanding of the effect of slag layers on the heat transfer characteristics of a coal fired MHD generator. Murty and Raju [7] studied a two-phase magnetohydrodynamic steady flow and heat transfer through a horizontal channel consisting of two parallel porous walls subject to the action of a uniform magnetic field, applied in the direction normal to the plane of flow.

All the above studies dealt with the flow formation under steady state operating conditions. However, there are many problems of practical interest that deal with a two-phase flow under time dependent operating conditions. In view of this, Umavathi et al. [8, 9] presented analytical solutions of an unsteady/oscillatory Hartmann two-fluid flow and heat transfer in a horizontal channel. An unsteady MHD flow of two-phase flow with time-dependent oscillatory wall transpiration velocity through a horizontal channel was investigated by Umavathi et al. [10]. Raju and Nagavalli [11] analysed the unsteady magnetohydrodynamic (MHD) two-layered fluids flow and heat transfer in a horizontal channel between two parallel plates in the presence of an applied magnetic and electric field, when the whole system is rotating about an axis perpendicular to the flow in the presence of an applied magnetic field. The objective of the present work is to present a semi-analytical solution of an unsteady Hartmann two-phase flow between two parallel plates in the presence of an electric and magnetic field. Both phases are incompressible and the flow is assumed to be fully-developed and one-dimensional time dependent due to a sudden application of a common pressure gradient in both phases. One of the fluids is assumed to be electrically conducting while the other fluid and the plates are assumed to be electrically non-conducting.

\section{Mathematical formulation}

The physical model shown in Fig. 1 consists of two infinite parallel plates extending in the $x$ - and $z$ -direction.

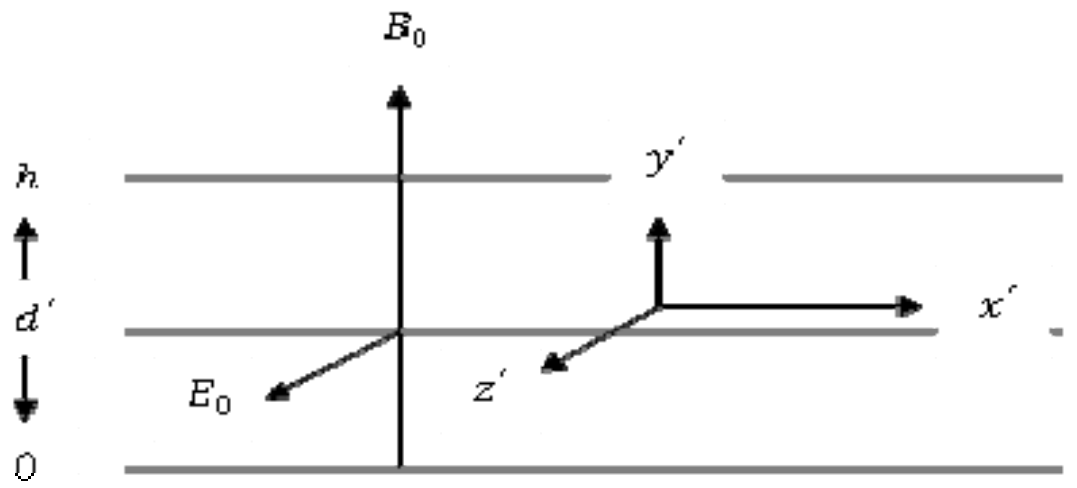

Fig.1. Physical configuration.

The region $0 \leq y^{\prime} \leq d^{\prime}$ is occupied by a fluid of viscosity $\mu_{l}$ and electrical conductivity $\sigma$, and the region $d^{\prime} \leq y^{\prime} \leq h$ is occupied by a layer of a non-conducting fluid of viscosity $\mu_{2}$. A constant magnetic field of strength $B_{0}$ is applied in the $y^{\prime}$-direction and a constant electric field $E_{0}$ is applied in $z$-direction. The flow is assumed to be time-dependent due to a sudden application of a common constant pressure gradient in both phases. Under the above stated assumptions, the dimensional equations of motion and the corresponding initial boundary and interface conditions for the two phases are

$$
\frac{\partial u_{1}^{\prime}}{\partial t^{\prime}}=v_{1} \frac{\partial^{2} u_{1}^{\prime}}{\partial y^{\prime 2}}-\frac{\partial p^{\prime}}{\partial x^{\prime}} \frac{1}{\rho_{l}}-\frac{\sigma B_{0}^{2}}{\rho_{l}}\left[u_{1}^{\prime}+\frac{E_{0}}{B_{0}}\right]
$$




$$
\begin{aligned}
& \frac{\partial u_{2}^{\prime}}{\partial t^{\prime}}=v_{2} \frac{\partial^{2} u_{2}^{\prime}}{\partial y^{\prime 2}}-\frac{\partial p^{\prime}}{\partial x^{\prime}} \frac{1}{\rho_{1}}, \\
& t^{\prime} \leq 0: u_{1}^{\prime}=u_{2}^{\prime}=0 \quad \text { for } \quad 0 \leq y^{\prime} \leq h, \\
& t^{\prime}>0:\left\{\begin{array}{l}
u_{1}^{\prime}(0)=0 \\
u_{2}^{\prime}(h)=0
\end{array}\right. \\
& t^{\prime}>0: y^{\prime}=d^{\prime}:\left\{\begin{array}{c}
\mu_{1} \frac{\partial u_{1}^{\prime}}{\partial y^{\prime}}=\mu_{2} \frac{\partial u_{2}^{\prime}}{\partial y^{\prime}} . \\
u_{1}^{\prime}=u_{2}^{\prime}
\end{array}\right.
\end{aligned}
$$

It is now convenient to non-dimensionalise the above equations by using the following dimensionless quantities

$$
\begin{aligned}
& Y=\frac{y^{\prime}}{h}, \quad\left(U_{1} ; U_{2}\right)=\left(u_{1}^{\prime} ; u_{2}^{\prime}\right)\left(\frac{h}{\mathrm{v}_{1}}\right), \quad T=\frac{t^{\prime} \mathrm{v}_{1}}{h^{2}}, \quad M^{2}=\frac{\sigma B_{0}^{2} h^{2}}{\rho_{1} \mathrm{v}_{1}}, \\
& d=\frac{d^{\prime}}{h}, \quad \alpha=\frac{\rho_{1}}{\rho_{2}}, \quad \delta=\frac{\mathrm{v}_{2}}{\mathrm{v}_{1}}, \quad P=-\frac{\partial p^{\prime}}{\partial x^{\prime}} \frac{1}{\rho_{1}} \frac{h^{3}}{\mathrm{v}_{1}^{\prime}}, \quad E=\frac{E_{0} h}{\mathrm{v}_{1} B_{0}}
\end{aligned}
$$

where the subscripts 1 and 2 refer to the lower and upper phases, respectively. $d$ is the dimensionless interface distance, $\alpha, \delta$ are the ratios of densities and kinematics viscosities of the two fluids, $T$ is the dimensionless time and $M$ is the Hartman number, which is a measure of the strength of the applied magnetic field and $E$ is the electric load parameter. Therefore, Eqs (2.1) - (2.3), respectively, become

$$
\begin{aligned}
& \frac{\partial U_{1}}{\partial T}=\frac{\partial^{2} U_{1}}{\partial Y^{2}}+P-M^{2}\left[U_{1}+E\right], \\
& \frac{\partial U_{2}}{\partial T}=\delta \frac{\partial^{2} U_{2}}{\partial Y^{2}}+\alpha P, \\
& T>0:\left\{\begin{array}{l}
U_{1}(0)=0 \\
U_{2}(1)=0
\end{array} \quad Y=d:\left\{\begin{array}{l}
\frac{\partial U_{1}}{\partial Y}=\frac{\delta}{\alpha} \frac{\partial U_{2}}{\partial Y^{\prime}} \\
U_{1}=U_{2}=U_{I}
\end{array}\right.\right.
\end{aligned}
$$

Taking the Laplace transform of Eqs (2.5) and (2.6) together with Eqs (2.7), we obtain the following ordinary differential equations, initial boundary and interface conditions 


$$
\begin{aligned}
& \frac{d^{2} \bar{U}_{1}}{d Y^{2}}-\left(M^{2}+s\right) \bar{U}_{1}=\frac{1}{s}\left[M^{2} E-P\right], \\
& \frac{d^{2} \bar{U}_{2}}{d Y^{2}}-\frac{s}{\delta} \bar{U}_{2}=-\frac{\alpha}{\delta} \frac{P}{s}, \\
& \bar{U}_{1}=\bar{U}_{2}=0,\left\{\begin{array}{l}
U_{1}(0)=0 \\
U_{2}(1)=0
\end{array} \text { and } Y=d:\left\{\begin{array}{l}
\frac{d \bar{U}_{1}}{d Y}=\frac{\delta}{\alpha} \frac{d \bar{U}_{2}}{d Y} \\
\bar{U}_{1}=\bar{U}_{2}=U_{I} s^{-1}
\end{array}\right.\right.
\end{aligned}
$$

where

$\bar{U}_{1}=\int_{0}^{\infty} U_{l}(Y, T) e^{-s T} d T, \bar{U}_{2}=\int_{0}^{\infty} U_{2}(Y, T) e^{-s T} d T, s>0$, where $s$ is the Laplace parameter.

The solution of Eqs (2.8) and (2.9) considering (2.10) are obtained respectively for $\bar{U}_{1}$ and $\bar{U}_{2}$ as follows

$$
\begin{aligned}
& \bar{U}_{1}=\frac{U_{I}}{s} \frac{\sinh \left(\delta_{1} Y\right)}{\sinh \left(\delta_{1} d\right)}+\frac{M^{2} E-P}{s \delta_{1}^{2}}\left[\frac{\sinh \left(\delta_{1}(d-Y)\right)}{\sinh \left(d \delta_{1}\right)}+\frac{\sinh \left(\delta_{1} Y\right)}{\sinh \left(\delta_{1} d\right)}-1\right], \\
& \bar{U}_{2}=\frac{\sinh \left(\delta_{2}(Y-1)\right)}{\sinh \left(\delta_{2}(d-l)\right)}\left[\frac{U_{I}}{s}+\frac{\alpha P}{s^{2}}\left\{\frac{\left.\cosh d \delta_{2}\right)}{\cosh \left(\delta_{2}\right)}-1\right\}\right]+\frac{P \alpha}{s^{2}}\left[1-\frac{\cosh \left(\delta_{2} Y\right)}{\cosh \left(\delta_{2}\right)}\right]
\end{aligned}
$$

where $U_{I}$ is the interface velocity, $\delta_{1}=\sqrt{M^{2}+s}, \delta_{2}=\sqrt{\frac{s}{\delta}}, \delta_{3}=\sqrt{\frac{\delta}{s}}$

$$
U_{I}=\delta_{3} \frac{P}{S}\left[\frac{\sinh \left(d \delta_{2}\right)}{\cosh \left(d \delta_{2}\right)}-\frac{\cosh \left(\delta_{3}(d-1)\right)}{\sinh \left(\delta_{3}(d-1)\right)}\left\{\frac{\cosh \left(d \delta_{2}\right)}{\cosh \left(\delta_{2}\right)}-1\right\}\right]+\frac{P-M^{2} E}{s \delta_{1}{ }^{2}}\left[\frac{1}{\sinh \left(d \delta_{1}\right)}-\frac{\cosh (d \gamma)}{\sinh (d \gamma)}\right]
$$

Equations (2.11) and (2.12) are inverted in terms of the Riemann - sum approximation [12] as

$$
\begin{aligned}
& U_{1}(Y, T)=\frac{\exp (\epsilon T)}{T}\left[\frac{1}{2} \bar{U}_{1}(Y, \epsilon)+\operatorname{Re} \sum_{k=1}^{N} \bar{U}_{1}\left(Y, \epsilon+\frac{i k \pi}{T}\right)(-1)^{k}\right], \\
& U_{2}(Y, T)=\frac{\exp (\epsilon T)}{T}\left[\frac{1}{2} \bar{U}_{2}(Y, \epsilon)+\operatorname{Re} \sum_{k=1}^{N} \bar{U}_{2}\left(Y, \epsilon+\frac{i k \pi}{T}\right)(-1)^{k}\right]
\end{aligned}
$$

where Re refers to the 'real part of', $i=\sqrt{-1}$ is an imaginary number, $N$ is the number of terms used in the Riemann-sum approximation and $\in$ is the real part of the Bromwich contour that is used in inverting Laplace transforms. The Riemann-sum approximation for the Laplace inversion involves a single summation for the numerical process. Its accuracy depends on the value of $\in$ and the truncation error dictated by $N$. According to Tzou [12], the value of $\in$ must be selected so that the Bromwich contour encloses all the 
branch point. For a faster convergence the quantity $\in T=4.7$ gives the most satisfactory results since other tested values of $\in T$ seem to need a longer computational time.

\subsection{Skin-friction and mass flux}

The skin friction $\tau_{0}$ at the lower channel wall $(Y=0)$ and $\tau_{1}$ at the upper channel wall $(Y=1)$ in terms of the Laplace parameter $s$ is computed by differentiating Eqs (2.11) and (2.12) respectively. Similarly, the mass flux in terms of the Laplace parameter $s, Q(Y, s)$ is obtained by adding the integrals of Eqs (2.11) and (2.12). The expressions are given by

$$
\begin{aligned}
& \left.\frac{d \bar{U}_{1}}{d Y}\right|_{Y=0}=c_{2} \delta_{2} . \\
& \left.\frac{d \bar{U}_{2}}{d Y}\right|_{Y=1}=\delta_{2}\left[c_{3} \sinh \left(\delta_{3}\right)+c_{4} \cosh \left(\delta_{3}\right)\right], \\
& Q=\int_{0}^{d} \bar{U}_{1}(Y, s) d Y+\int_{d}^{1} \bar{U}_{2}(Y, s) d Y=\frac{1}{\gamma}\left[c_{1} \sinh \left(d \delta_{1}\right)+c_{2}\left\{\cosh \left(d \delta_{1}\right)-1\right\}\right]+ \\
& +\delta_{3}\left[c_{3} \sinh \left(\delta_{2}\right)+c_{4}\left\{\cosh \left(\delta_{2}\right)-\cosh \left(\delta_{2} d\right)\right\}\right]+\frac{P d}{s \gamma^{2}}+\frac{P \alpha}{s^{2}}(1-d) \\
& c_{1}=\frac{P-M^{2} E}{s \delta_{1}^{2}}, \\
& \left.c_{3}=\left[\frac{U_{I}}{s}+\frac{\alpha P}{s^{2}}\left\{\frac{\cosh \left(d \delta_{2}\right)}{\sinh \left(\delta_{2}\right)}-1\right\}\right] \frac{U_{I}}{s}-\frac{P-M^{2} E}{s \delta_{1}^{2}}\right] \frac{1}{\sinh \left(d \delta_{1}\right)}+\frac{P-M^{2} E}{s \delta_{1}^{2}} \frac{\cosh \left(d \delta_{1}\right)}{\sinh \left(d \delta_{1}\right)}, \\
& c_{4}=\left[\frac{U_{I}}{s}+\frac{\alpha P}{s^{2}}\left\{\frac{\cosh \left(d \delta_{2}\right)}{\sinh \left(\delta_{2}\right)}-1\right\}\right] \frac{\sinh \left(\delta_{2}\right)}{\sinh \left(\delta_{2}(d-1)\right)} \cdot \frac{P \alpha}{s^{2} \cosh \left(\delta_{2}\right)},
\end{aligned}
$$

where

\subsection{Validation of the method}

In order to validate the accuracy of the Riemann-sum approximation method, we set out to find the solution of the steady state mathematical model, which should coincide with the transient solution at large time. The equations for the steady state velocities $\left(U_{s 1}, U_{s 2}\right)$ for the two phases are obtained by setting $\frac{\partial()}{\partial T}$ in Eqs (2.5) and (2.6) to zero. Then the following equations result

$$
\begin{aligned}
& \frac{d^{2} U_{s 1}}{d Y^{2}}-M^{2} U_{s 1}=M^{2} E-P, \\
& \frac{d^{2} U_{s 2}}{d Y^{2}}=-\frac{\alpha P}{\delta} .
\end{aligned}
$$


The boundary and interface conditions are

$$
\begin{aligned}
& U_{s 1}(0)=0 ; \quad U_{s 2}(1)=0 . \\
& Y=d:\left\{\begin{array}{c}
\frac{\partial U_{s 1}}{\partial Y}=\frac{\delta}{\alpha} \frac{\partial U_{s 2}}{\partial Y^{2}} . \\
U_{s 1}=U_{s 2}
\end{array}\right.
\end{aligned}
$$

The solution of Eqs (2.19) and (2.20) considering (2.21) which give the steady state velocities for the two phases $U_{s 1}$ and $U_{s 2}$ are given as

$$
\begin{aligned}
& U_{s 1}(Y)=\frac{U_{s 1} \sinh (M Y)}{\sinh (M d)}+\frac{P-M^{2} E}{M^{2}}+\left[\frac{M^{2} E-P}{M^{2}}\right] \frac{\sinh (M(d-Y))}{\sinh (M d)}, \\
& \boldsymbol{U}_{\boldsymbol{s} 2}(\boldsymbol{Y})=\frac{\boldsymbol{U}_{\boldsymbol{s} 1}(\boldsymbol{Y}-1)}{(\boldsymbol{d}-1)}+\frac{\boldsymbol{P} \boldsymbol{\alpha}}{2 \boldsymbol{\delta}}\left\{(\boldsymbol{Y}-1) \boldsymbol{d}+\left(\boldsymbol{Y}-\boldsymbol{Y}^{2}\right)\right\} .
\end{aligned}
$$

The steady state skin-friction at the lower channel wall $\left(\tau_{0}\right)$ and upper channel wall $\left(\tau_{1}\right)$ are

$$
\begin{aligned}
& \left.\frac{d U_{s 1}}{d Y}\right|_{Y=0}=\frac{\left(P-M^{2} E\right)[\cosh (M d)-1]}{M \sinh (M d)}+\frac{M U_{s I}}{\sinh (M d)}, \\
& \left.\frac{d U_{s 2}}{d Y}\right|_{Y=1}=\frac{U_{s I}}{(d-l)}+\frac{P \alpha}{2 \delta}(d-l) .
\end{aligned}
$$

The steady state mass flux is given as

$$
\begin{aligned}
& Q_{s}=\int_{0}^{d} U_{s 1}(y) d Y+\int_{d}^{1} U_{s 2}(Y) d Y=\frac{M^{2} E-P}{M^{2}}\left[\frac{2 \cosh ((M d)-1)}{M \sinh (M d)}-d\right]+\frac{U_{s I}[\cosh (M d)-1]}{M \sinh (M d)}+ \\
& +\frac{U_{s I}\left[\frac{1-d^{2}}{2}-(1-d)\right]}{(d-1)}+\frac{P \alpha\left[d\left\{\frac{1-d^{2}}{2}-(1-d)\right\}\right]}{2 \delta}+\frac{P \alpha\left[\frac{1-d^{2}}{2}-\frac{1-d^{2}}{3}\right]}{2 \delta}
\end{aligned}
$$

where

$$
U_{s I}=\frac{1}{F}\left[\frac{1}{2}(1-d)-\frac{P}{M}\left(\frac{1}{\sinh (M d)}-\frac{\cosh (M d)}{\sinh (M d)}\right)\right], \quad F=\frac{M \cosh (M d)}{\sinh (M d)}-\frac{\delta}{\alpha} \frac{1}{d-1}
$$

\subsection{Numerical solution}

In this section, we have used the implicit finite difference method to ascertain the correctness of the Riemann-sum approximation method. The procedure we have adopted involves dividing the solutions into grid points and approximating the differential equations by the finite difference equations and then solving 
the difference equations subject to the prescribed initial, boundary and interface conditions. Thus Eqs (2.5) and (2.6) become

$$
\begin{aligned}
& \frac{U_{1}(i, j)-U_{1}(i, j-1)}{\Delta T}=\frac{U_{1}(i+1, j)-2 U_{1}(i, j)+U_{1}(i-1, j)}{\Delta Y^{2}}+P-M^{2}\left[U_{1}(i, j)+E\right], \\
& \frac{U_{2}(i, j)-U_{2}(i, j-1)}{\Delta T}=\delta \frac{\left[U_{2}(i+1, j)-2 U_{2}(i, j)+U_{2}(i-1, j)\right]}{\Delta Y^{2}}+P \alpha .
\end{aligned}
$$

The index $i$ refers to $Y$ and $j$ refers to $T$. The time derivative is replaced by the backward difference formula, while the spatial derivatives are replaced by the central difference formula. The above equations are solved by Thomas algorithm by manipulating into a system of linear algebraic equations in the tridiagonal form. At each time step, the process of numerical integration for every dependent variable starts from the first neighbouring grid point of the lower plate at $Y=0$ and proceeds towards the upper plate at $Y=1$. The process of computation is advanced until a steady state is approached by satisfying the following convergence criterion

$$
\frac{\sum\left|A_{i, j+1}-A_{j}\right|}{M^{*}|M|_{\max }} \leq 10^{-6},
$$

with respect to the fluids velocity. Here $A_{(i, j)}$ stands for the velocity field in each phase. $M^{*}$ is the number of interior grid points and $|M|_{\max }$ is the maximum absolute value of $A_{(i, j)}$. In the numerical computation special attention is needed to specify $\Delta T$ to get a steady state solution as rapid as possible, yet small enough to avoid instabilities. It is set, which is suitable for the present computation, as

$$
\Delta T=\operatorname{stabr} \times(\Delta Y)^{2} .
$$

The parameter stabr is determined by numerical experimentation in order to achieve convergence and stability of the solution procedure. Numerical experiments show that the value 2 is suitable for the present numerical computation. The numerical values obtained through the numerical solution, Riemannsum approximation and exact steady state solution are in excellent agreement at large value of time $(T \leq 0.8)$ as shown in Tab.1.

\section{Results and discussion}

In order to have a physical insight into the problem, semi-analytical solutions for momentum equations in both phases are obtained using a combination of the Laplace transform technique and the Riemann-sum approximation approach. The results are shown graphically in Figs 2 - 10 to elucidate the effects of dimensionless time $(t)$, Hartmann number $(\mathrm{M})$, ratios of viscosities $(\delta)$, ratios of densities $(\alpha)$ and dimensional interface distance $d$ for $P=+5$ or -5 . The considered values of the electric load parameter $(E)$ are $-1,0$ and 1 , where $E=0$, represents the short-circuited case. Of particular importance in the analysis is the case when $E$ is different from zero indicating that the value of $E$ defines the system as generator, flow meter or pump.

Figures 2 and 3 show the effect of dimensionless time $(t)$ on the velocity profiles. From Figs 2, the velocity increases as $t$ increases and finally attains its steady state value for the considered values of controlling parameters. 


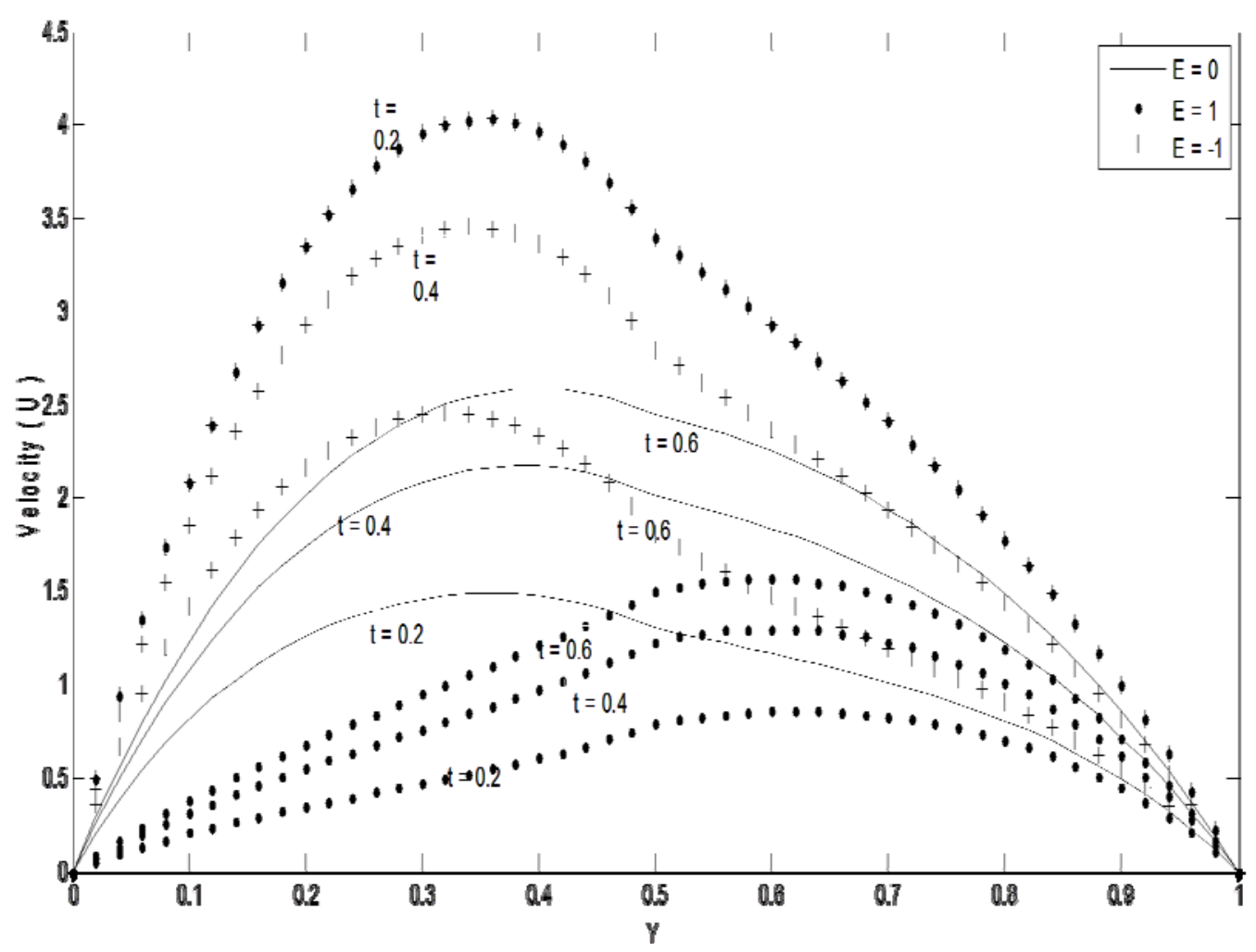

Fig.2. Velocity profiles for different values of time $t[d=0.5, \alpha=0.5, \delta=1.0, M=2.0$ and $P=5.0]$.

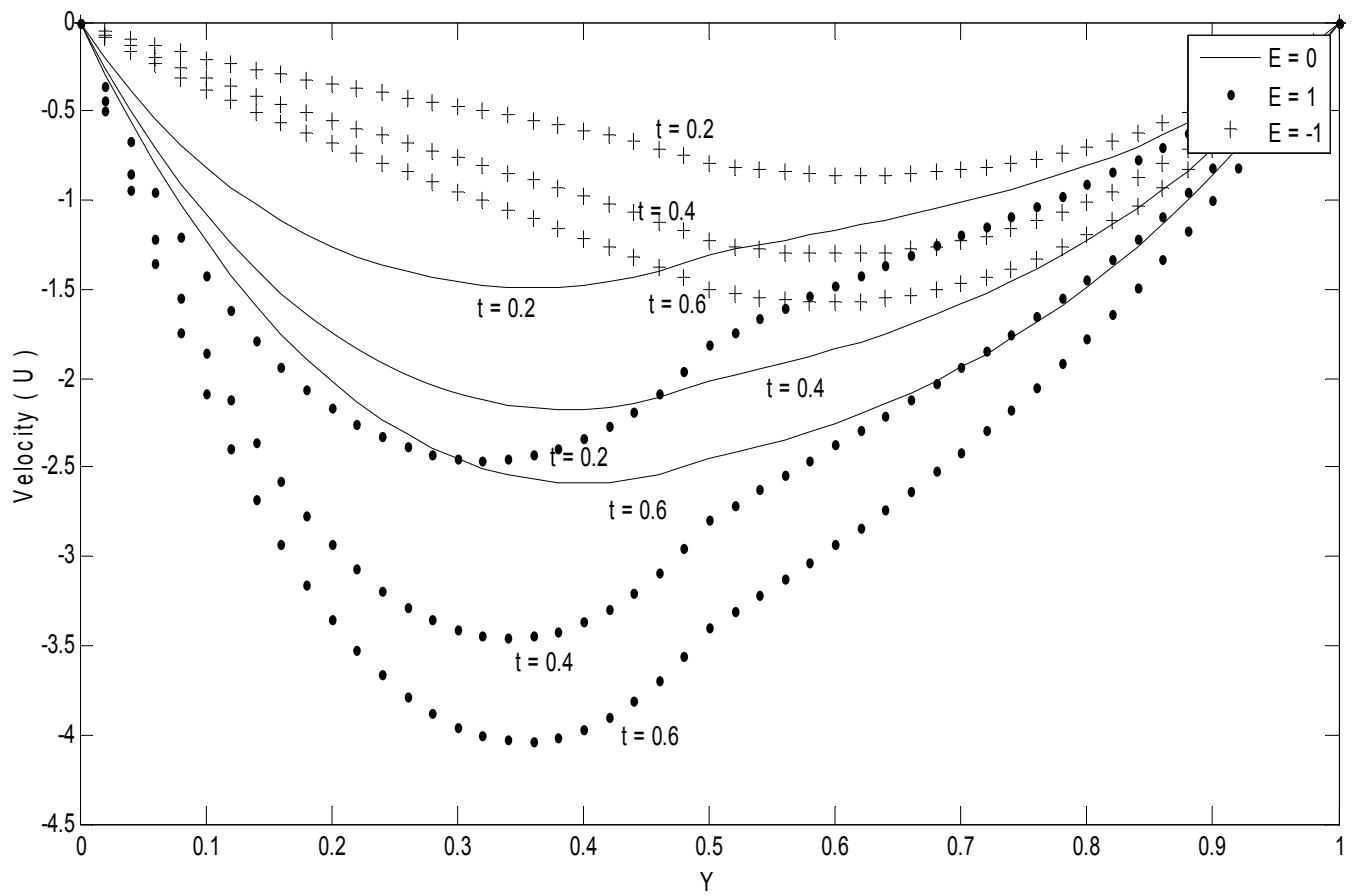

Fig.3. Velocity profiles for different values of time $t[d=0.5, \alpha=0.5, \delta=1.0, M=2.0$ and $P=-5.0]$. 
From Fig. 3 it is clear that a reverse flow occurs due to a sudden application of adverse applied pressure gradient $(P=-5.0)$. Furthermore, from Fig.3 we conclude that the reverse flow could be controlled by considering suitable values of the electric load parameter $(E)$. Figures 4 and 5 depict the effect of the Hartmann number (M) for positive as well as negative value of $P$, respectively. It is observed that when $E=0$, the magnitude of velocity decreases as $\mathrm{M}$ increases. Moreover, when $E=-1.0$ and $E=+1$, the magnitude of velocity increases as $M$ increases. Figure 5 illustrates that in the absence of the electric load parameter the reverse flow could be controlled by increasing the Hartman number. The effect of the kinematic viscosity ratio on the velocity is shown in Figs 6 and 7. It is observed that as the viscosity ratio increases, the magnitude of velocity decreases for all considered values of the electric load parameter. Hence we conclude that velocity is higher when the kinematic viscosity of the non-conducting fluid is higher than the kinematic viscosity of the conducting fluid. The effects of the density ratio $(\alpha)$ on the flow behavior are presented in Figs 8 and 9. It is observed that as the density ratio increases magnitude of velocity increases. The effects of the dimensionless interface distance $d$ on velocity are depicted in Figs 10 and 11. It is observed that as the interface distance increases, the magnitude of velocity increases.

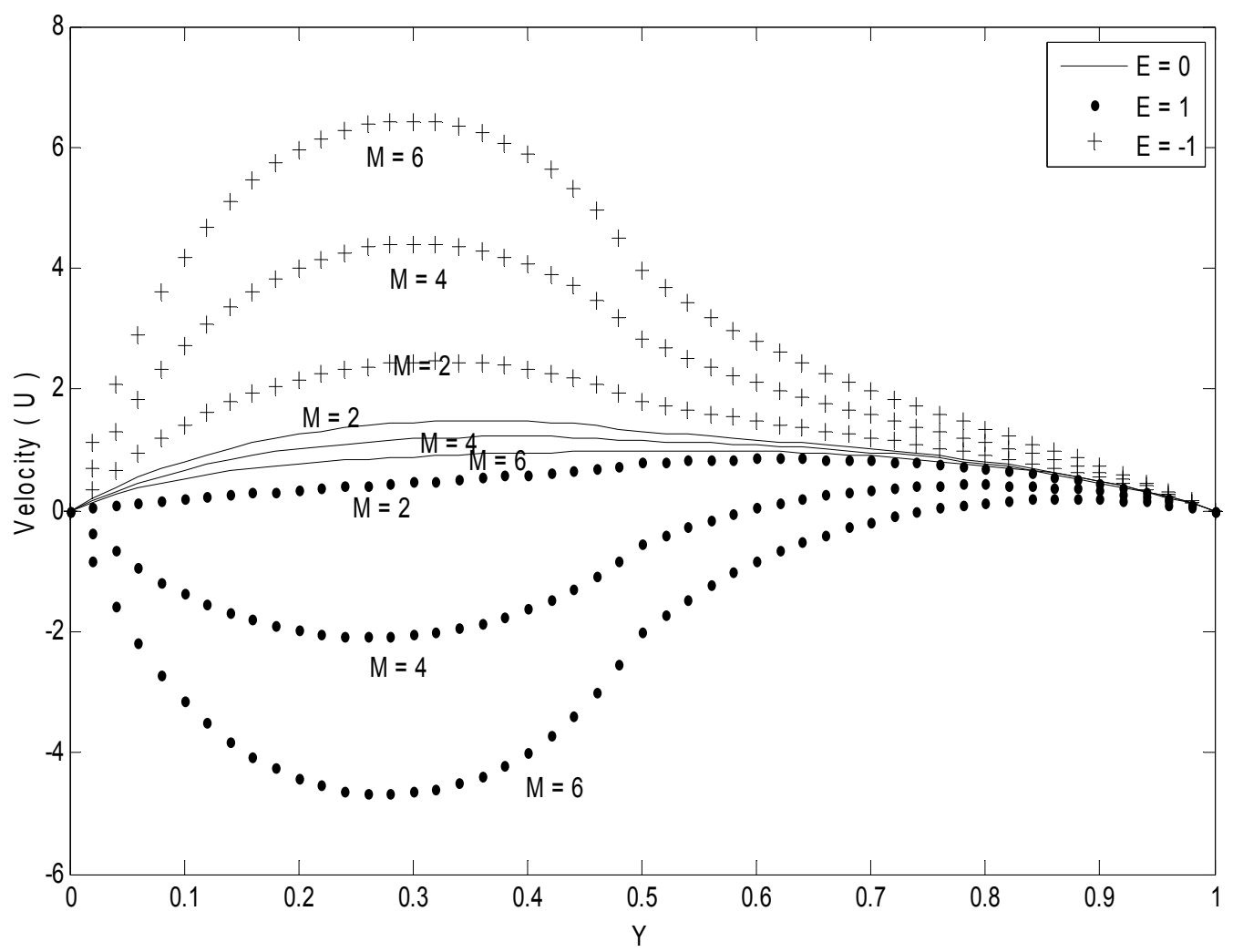

Fig.4. Velocity profiles for different values of $M[d=0.5, \alpha=0.5, \delta=1.0, t=0.2$ and $P=5.0]$. 


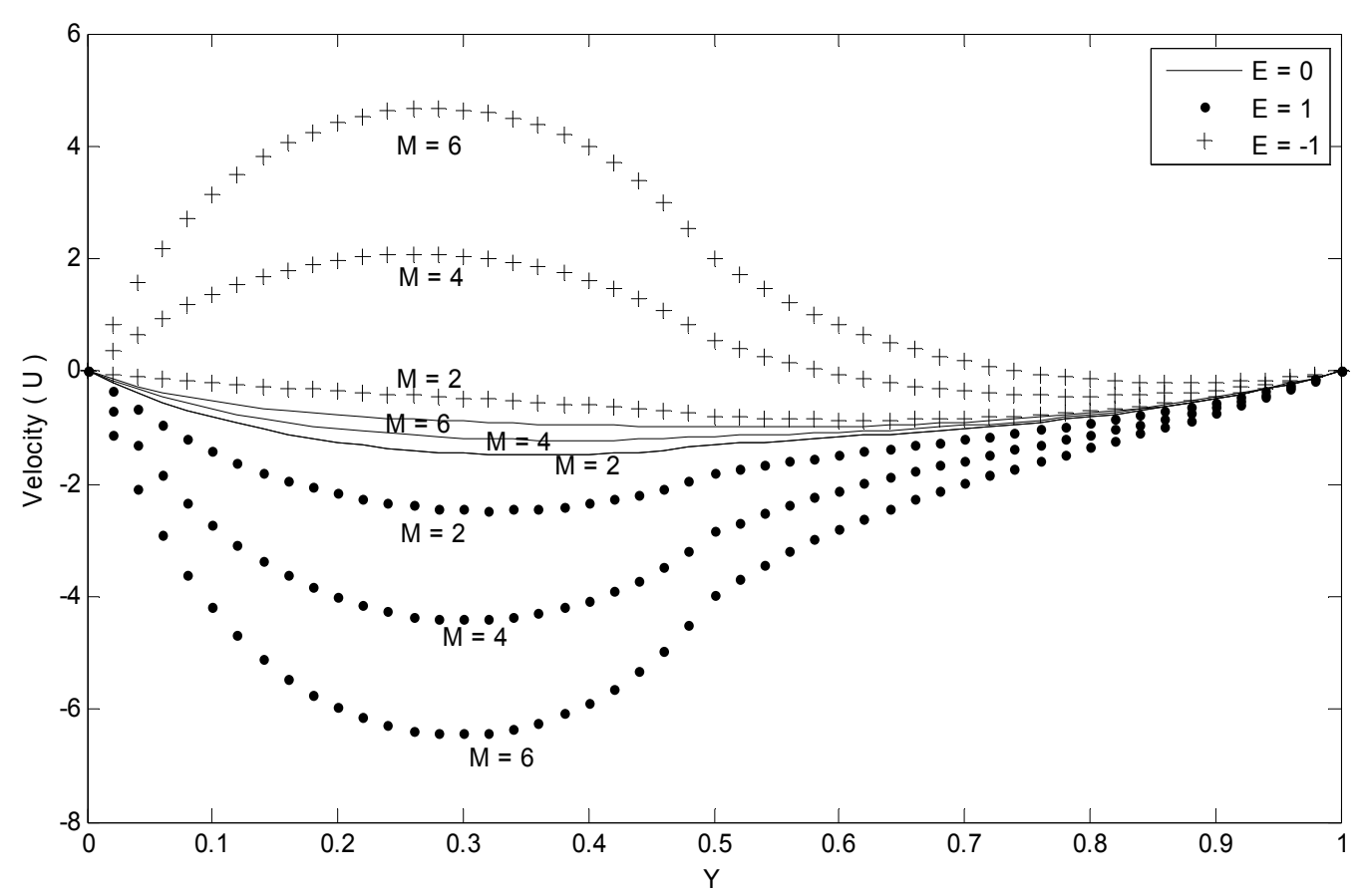

Fig.5. Velocity profiles for different values of $M[d=0.5, \alpha=0.5, \delta=1.0, t=0.2$ and $P=-5.0]$.

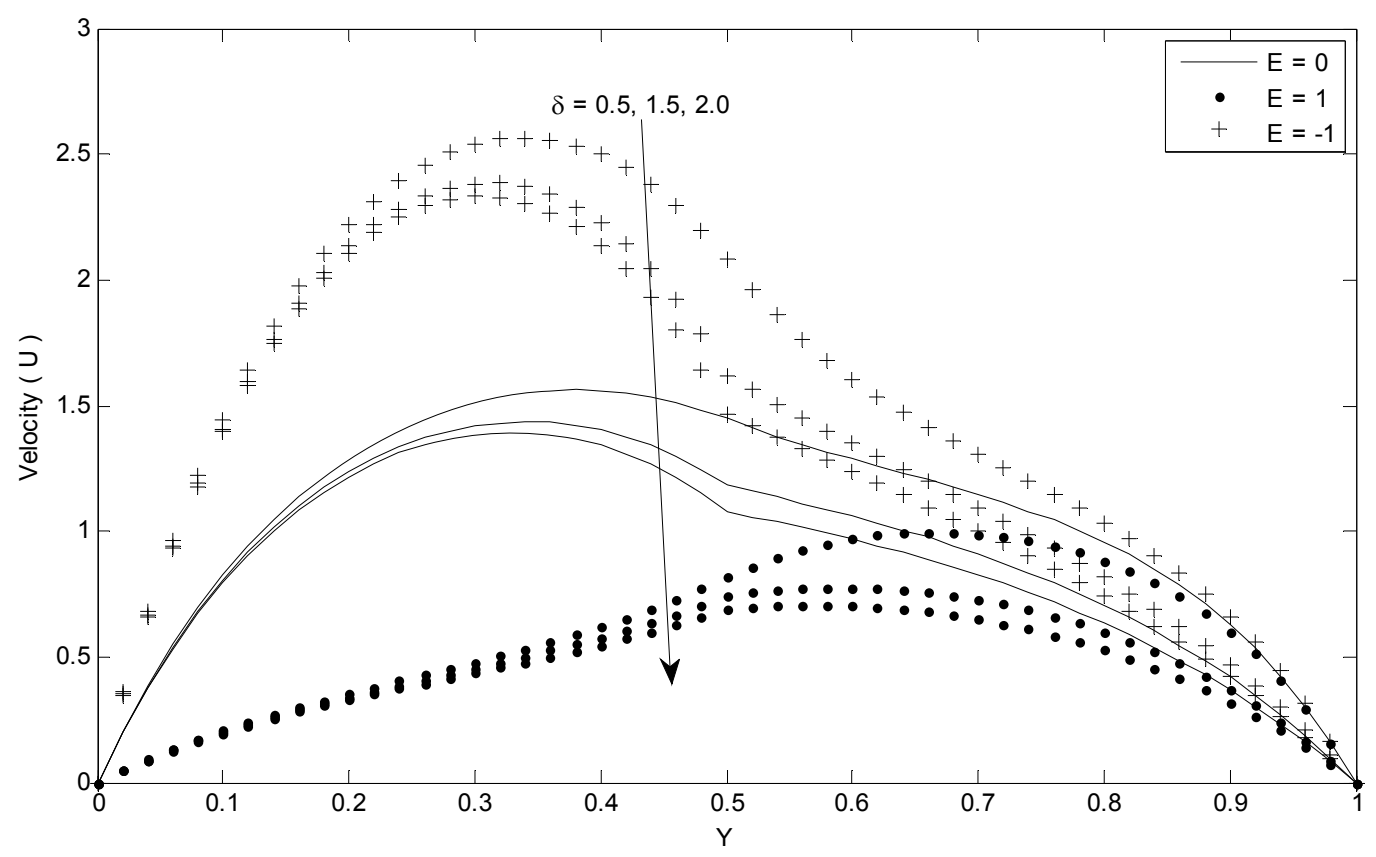

Fig.6. Velocity profiles for different values of $\delta[d=0.5, \alpha=0.5, M=2.0, t=0.2$ and $P=5.0]$. 


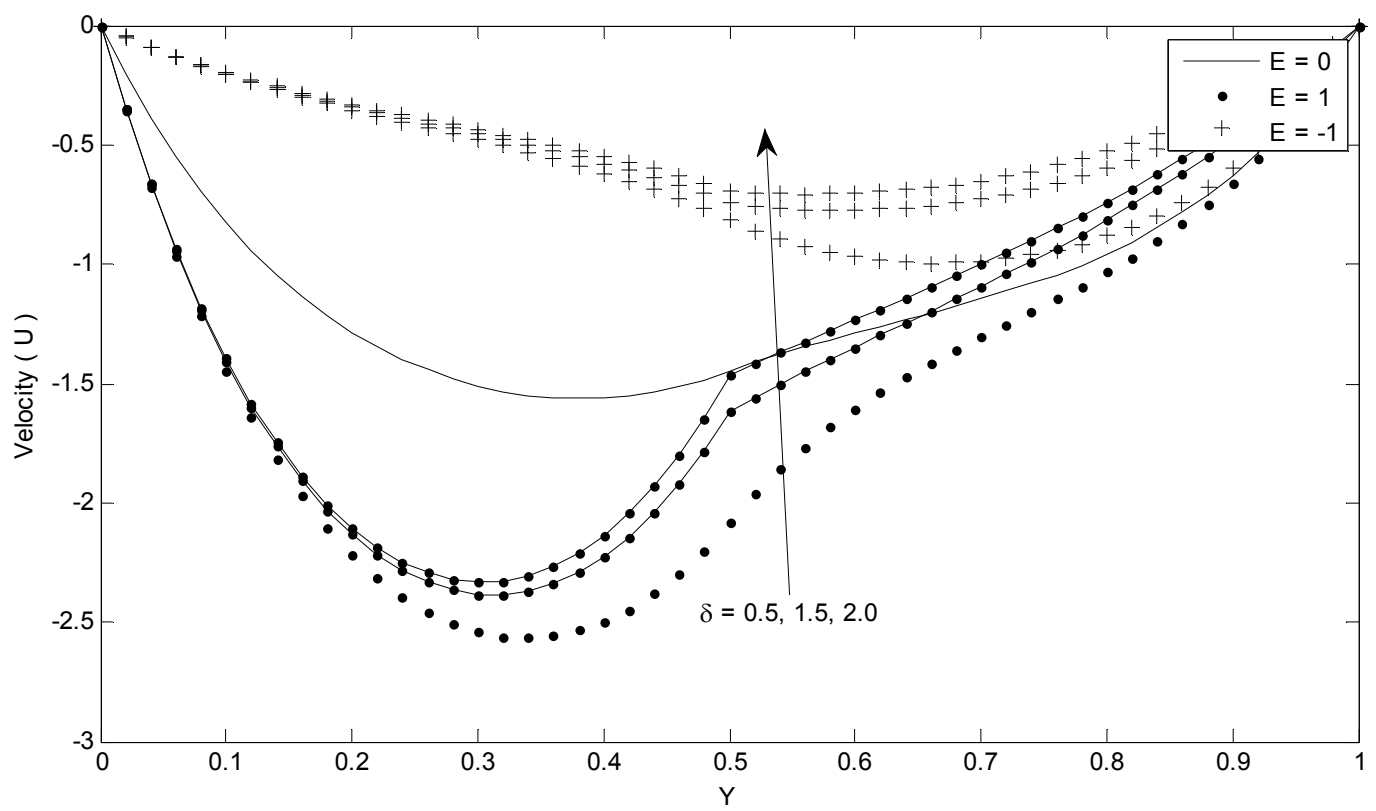

Fig.7. Velocity profiles for different values of $\delta[d=0.5, \alpha=0.5, M=2.0, t=0.2$ and $P=-5.0]$.

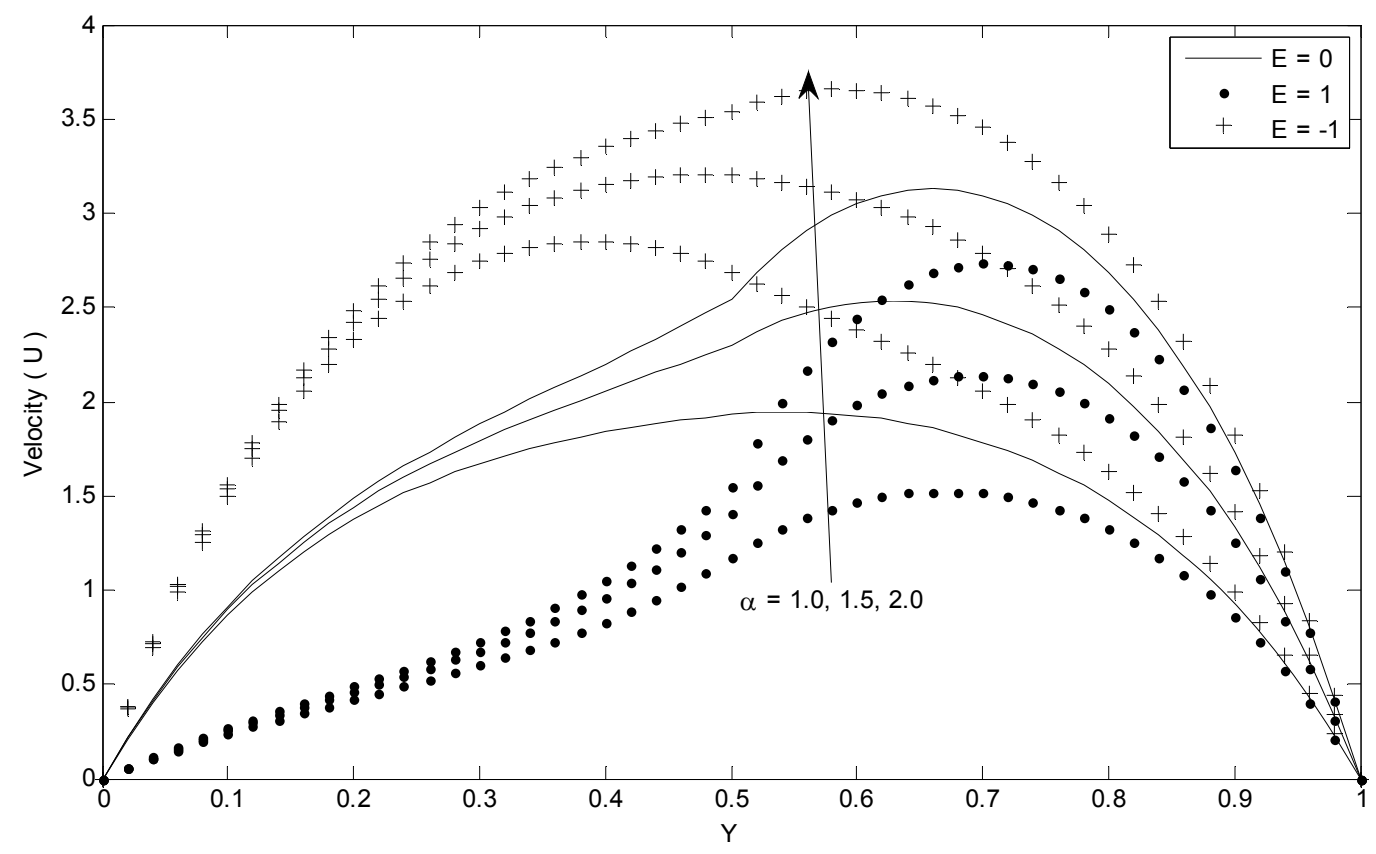

Fig.8. Velocity profiles for different values of $\alpha[d=0.5, \delta=1.0, M=2.0, t=0.2$ and $P=5.0]$. 


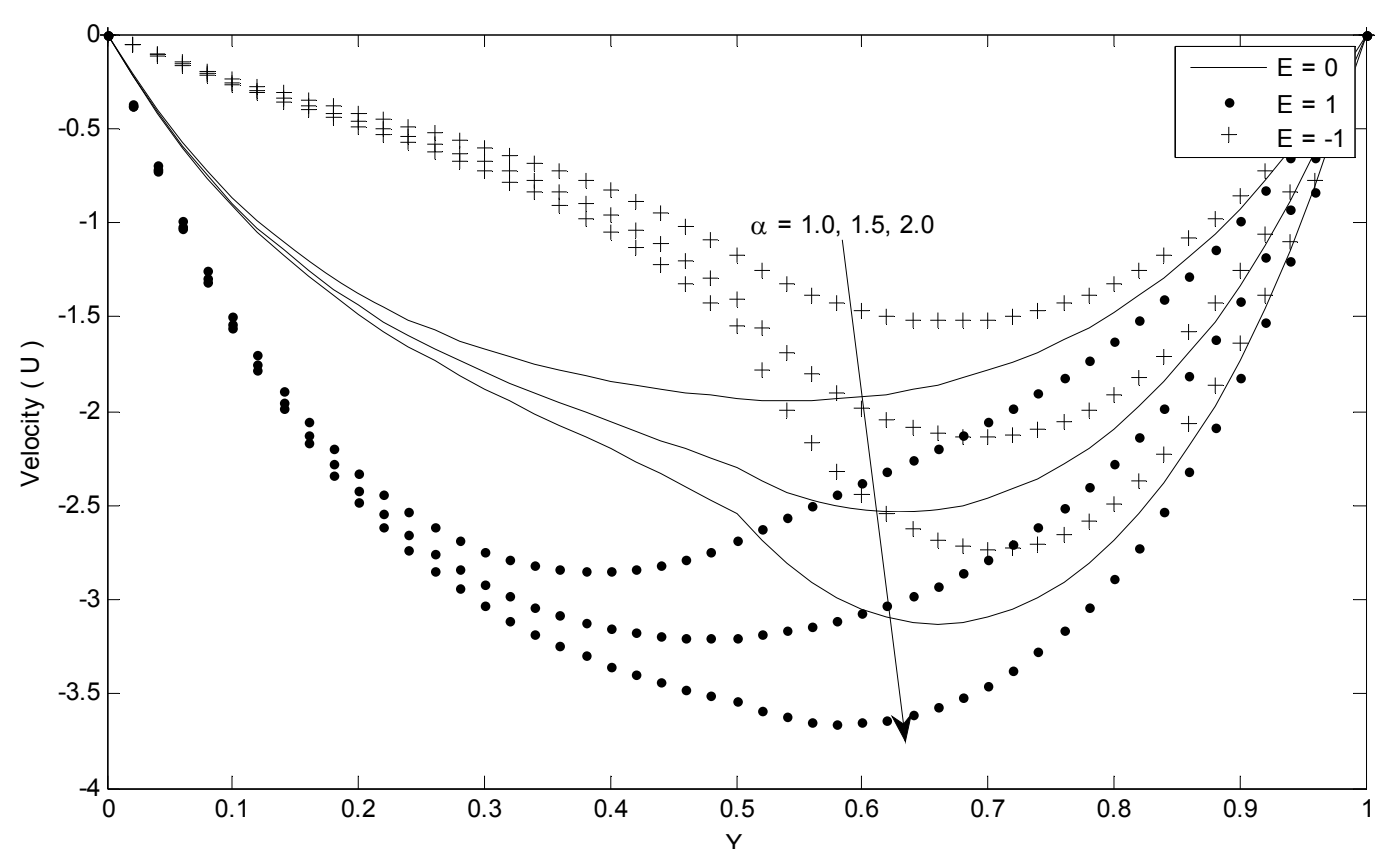

Fig.9. Velocity profiles for different values of $\alpha[d=0.5, \delta=1.0, M=2.0, t=0.2$ and $P=-5.0]$.

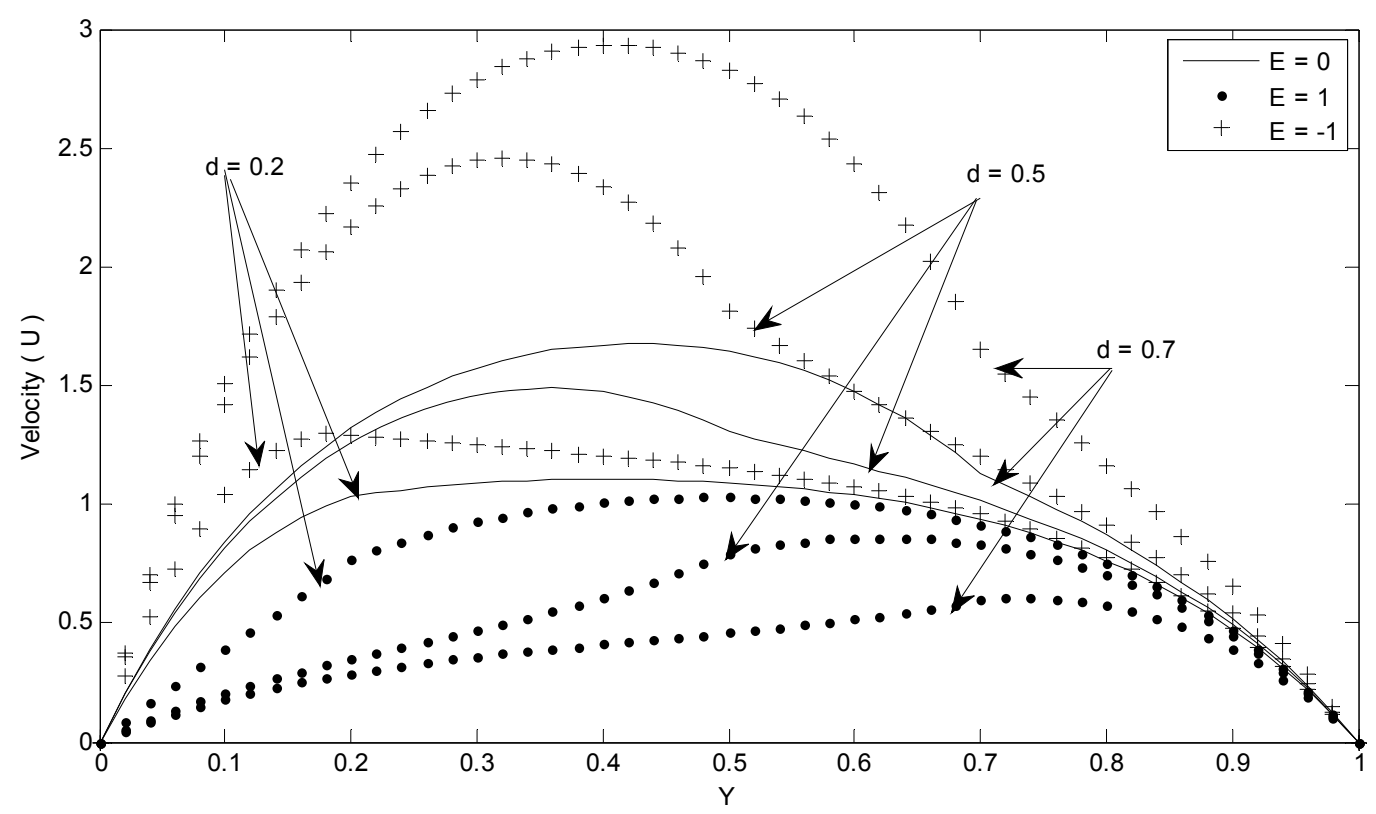

Fig.10. Velocity profiles for different values of $d[\alpha=0.5, \delta=1.0, M=2.0, t=0.2$ and $P=5.0]$. 


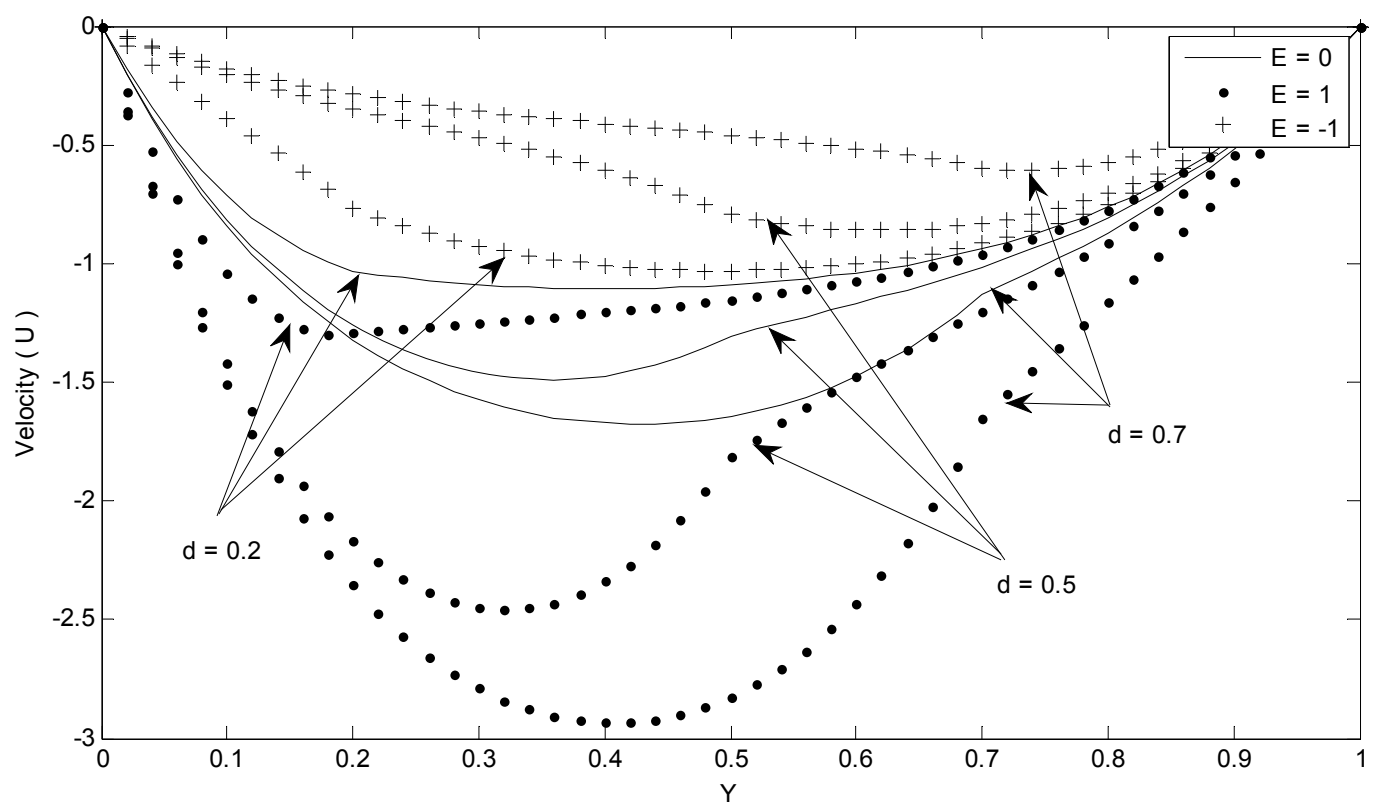

Fig.11. Velocity profiles for different values of $d[\alpha=0.5, \delta=1.0, M=2.0, t=0.2$ and $P=-5.0]$.

\section{Conclusions}

The time dependent Hartmann two-phase flow is studied semi-analytically. One of the fluids is assumed to be electrically conducting, while the other is electrically non-conducting. Separate closed form solutions for velocity of each phase are obtained taking into consideration suitable interface conditions. The results show that:

1. Higher velocity could be achieved by considering a higher value of the kinematic viscosity of the non-conducting fluid in comparison to the kinematic viscosity of the conducting fluid.

2. Reverse flow could be controlled by considering suitable values of the electric load parameter.

3. Velocity could be suppressed by considering a heavier non-conducting fluid in comparison to the conducting fluid.

\section{Nomenclature}

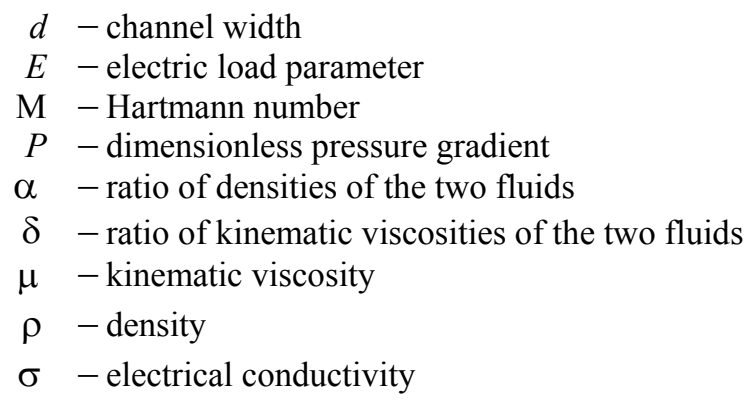

\section{Subscripts}

1 - lower phase

2 - upper phase

$I$ - interface 


\section{References}

[1] Shail R. (1973): On laminar two-phase flows in magnetohydrodynamics. - Int. J. Engng Sci., vol.11, pp.11031108.

[2] Rudraiah N., Ramaih B.K. and Rajashekhar B.M. (1975): Hartmann flow over a permeable bed. - Int. J. Eng. Sci., vol.13, pp.1-24.

[3] Hartmann J. and Lazarus F. (1937): Hg-Dynamics-II, Experimental investigations on the flow of Mercury in a homogeneous magnetic field. - Det Kgl. Danske Videnskabernes Selskab. Mathematisk-Fysiske Meddelelser., XV7.

[4] Hartmann J. (1937): Hg-Dynamics-I, theory of Laminar flow of an electrically conducting liquid in a homogeneous magnetic field. - Det Kgl. Danske Videnskabernes Selskab. Mathematisk-fysiske Meddelelser., XV6.

[5] Malashetty M.S. and Leela V. (1992): Magnetohydrodynamic heat transfer in two phase flow. - Int. J. Engng. Sci., vol.30, No.1, pp.371-377.

[6] Lohrasbi J. and Sahai V. (1988): Magnetohydrodynamic heat transfer in two-phase flow between parallel plates. Applied Scientific, Research, vol.45, pp.53-66.

[7] Murty P.S.R. and Raju T.L. (2014): MHD two-phase flow and heat transfer between two parallel porous walls in a rotating system. - British Journal of Mathematics and Computer Science, vol.4, No.13, pp.1894-1907.

[8] Umavathi J.C., Chamkha A.J., Mateen A. and Al-Mudhaf A. (2005): Unsteady two-fluid flow and heat transfer in a horizontal channel. - Heat and Mass Transfer, vol.422, pp.81-90.

[9] Umavathi J.C., Mateen A., Chamkha A.J. and Mudhaf A.A. (2006): Oscillatory Hartmann two-fluid flow and heat transfer in a horizontal channel. - International Journal of Applied Mechanics and Engineering, vol.11, No.1, pp.155-178.

[10] Umavathi J.C., Chamkha A.J., Mateen A. and Kumar J.P. (2008): Unsteady magnetohydrodynamic two-fluid flow and heat transfer in a horizontal channel. - Heat and Technology, vol.262, pp.121-133.

[11] Linga Raju T. and Nagavalli M. (2014): MHD two -layered unsteady fluid flow and heat transfer through a horizontal channel between parallel plates in a rotating system. - Int. J. of Applied Mechanics and Engineering, vol.19, No.1, pp.97-121.

[12] Jha B.K. and Apere C.A. (2010): Unsteady MHD Couette flow in an annuli: the Riemann-sum approximation approach. - J. Phys. Soc. Jpn., vol.79, 124403.

[13] Tzou D.Y. (1997): Macro to Microscale Heat Transfer: The Lagging Behaviour. - Taylor and Francis.

Received: September 19, 2015

Revised: June 28, 2016 